\title{
Ziehl Neelsen Acid Fast Staining Method
}

National Cancer Institute

\section{Source}

National Cancer Institute. Ziehl Neelsen Acid Fast Staining Method. NCI Thesaurus. Code C85831.

A microscopy acid fast staining method that utilizes Ziehl-Neelsen carbol fuchsin, acid alcohol and methylene blue, to determine the presence of acid fast microorganisms. This acid fast staining technique requires heating during the primary staining step involving the carbol fuchsin. 